\title{
La recherche agricole, vecteur de dynamiques collectives des territoires ruraux : I'Indication géographique «Vales da Uva Goethe » au Brésil
}

\author{
Claire Cerdan ${ }^{1,2, *}$, Natalia Andrade ${ }^{3}$, Aparecido Lima da Silva ${ }^{4}$, Hamilton Vieira ${ }^{4}$, Edson Silva ${ }^{4}$ \\ et Jean Louis Le Guerroué ${ }^{2,5}$ \\ ${ }^{1}$ CIRAD, UMR INNOVATION, 34398 Montpellier, France \\ ${ }^{2}$ Univ Montpellier, CIRAD, INRA, Montpellier SupAgro, Montpellier, France \\ ${ }^{3}$ Universidade Federal de Santa Catarina, Florianópolis, Brasil \\ ${ }^{4}$ Empresa de Pesquisa Agropecuária e Extensão Rural de Santa Catarina (EPAGRI), Florianópolis, Brasil \\ ${ }^{5}$ CIRAD, UMR ART-DEV, Universidade de Brasília, Brasília, Brasil
}

\begin{abstract}
Résumé - L'article analyse en quoi et comment la recherche peut être un vecteur de renforcement des dynamiques collectives des territoires par la méthode participative ImpresS (Impact des recherches au Sud). Celle-ci qualifie la façon dont la recherche accompagne les processus d'innovation et y contribue, et la façon dont elle renforce les actions collectives par la création de nouveaux espaces de dialogue et d'échanges entre les chercheurs et les parties prenantes d'un projet d'indication géographique (IG). La contribution de la recherche est analysée dans trois objets et échelles d'analyse : l'organisation porteuse de l'IG - l'association ProGoethe -; le territoire de l'IG «Vales da Uva Goethe»; et ses liens avec d'autres territoires impliqués dans des démarches de valorisation de produits locaux.
\end{abstract}

Mots clés : indication géographique / action collective / chemin de l'impact / territoire

\begin{abstract}
Agricultural research, a key factor in promoting collective dynamics in rural area: the "Vales da Uva Goethe" Geographical Indication (IG) in Brazil. The article analyses how the research may strengthen the collective dynamics in rural territories. The participatory method called ImpresS (Impact of Research in the South) qualifies how research contributes to innovation processes and strengthens collective actions by creating new spaces for dialogue and exchange based on shared values between local stakeholders and researchers. We analyse the research contribution focusing on three objects and scales of analysis: the GI organization ProGoethe, the GI "Vales da Uva Goethe" places and their interactions with other rural territories also involved with local products valorisation strategies.
\end{abstract}

Keywords: geographical indication / collective action / territory / impact pathways

\section{Introduction}

Les indications géographiques (IG) sont un des aspects des droits de propriété intellectuelle qui touchent au commerce (OMC, 1994). Elles protègent des produits associés, de par leurs caractéristiques, à une origine géographique spécifique. C'est d'abord un outil qui règle les droits d'usage des noms sur les marchés. Depuis 1994, les IG ont connu un essor important dans les pays du Sud, qui s'explique en partie par le mouvement général de réforme des politiques agricoles

\footnotetext{
*Auteur de correspondance : claire.cerdan@cirad.fr
}

(suppression de la réglementation publique des prix agricoles) et la définition de nouvelles politiques rurales (Allaire, 2009). Pour certains responsables politiques, les IG peuvent devenir un instrument de développement rural et de promotion de la biodiversité (Sylvander et al., 2011; Boisvert, 2013). Or, cela est discutable, toutes les IG n'ayant pas les mêmes effets selon la façon dont elles ont été construites. Ainsi, l'analyse du développement d'une IG doit toujours être contextualisée, les résultats observés étant potentiellement liés au dispositif d'enregistrement, aux acteurs en présence et aux politiques qui les portent (Allaire, 2009). Parmi ces acteurs, la recherche agricole joue parfois un rôle important. Mais les IG qui reconnaissent et protègent les pratiques de production et de 


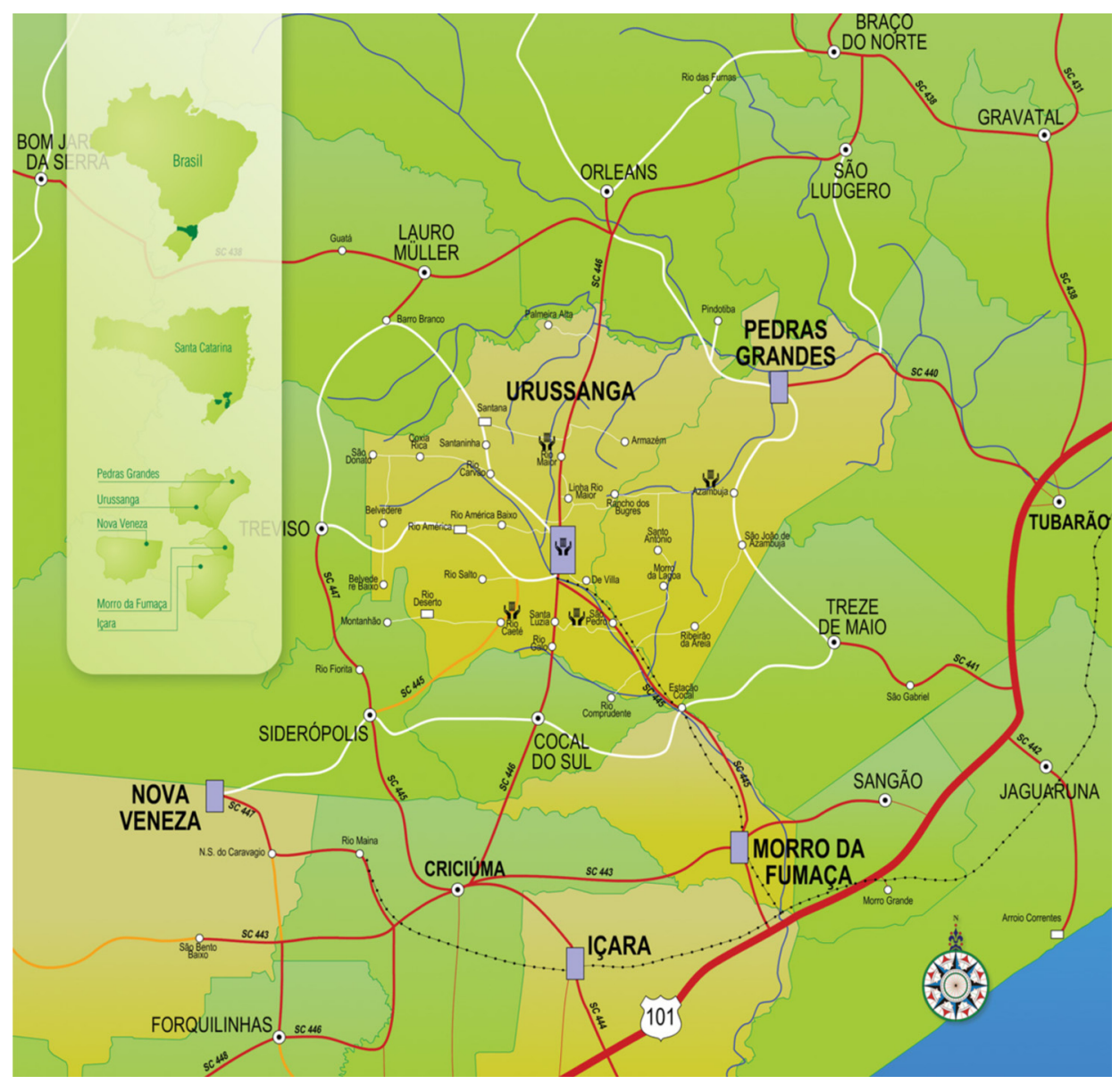

Fig. 1. Localisation et délimitation de la région d'étude. Source : Rebollar et al. 2007.

Fig. 1. Localization and delimitation of the area study.

consommation d'une région ne recourent pas forcément à des innovations techniques issues de travaux de recherche. En revanche, ce sont des initiatives collectives qui requièrent une coordination entre les acteurs d'un territoire pour préserver une réputation, un bien commun (Fournier, 2016; Torre et Wallet, 2016) et définir des règles de production et de contrôle (Vandecandelaere et al., 2010). En s'appuyant sur l'étude d'une IG au sud du Brésil, les vins des «Vales da Uva Goethe» (vallées du raisin Goethe), cet article analyse en quoi et comment la recherche peut être un vecteur de renforcement de ces dynamiques collectives.

Cultivé dans une région du sud du Brésil depuis la fin du $\mathrm{XIX}^{\mathrm{e}}$ siècle (Fig. 1), le cépage Goethe, sans reconnaissance de sa qualité spécifique, était en perte de vitesse au début des années 2000. Certaines parties du vignoble avaient été arrachées. Ce cépage, issu du croisement naturel de variétés américaines Vitis labrusca et européennes Vitis vinifera, était délaissé par la recherche et les connaisseurs à cause de son goût foxé (musqué). Les producteurs, attachés à ce cépage pour des raisons culturelles et historiques, étaient conscients que sa relance passait par la reconnaissance de son originalité, la mise en valeur de ses saveurs uniques et l'amélioration de la qualité des vins produits. Les producteurs connaissaient les défauts de leur vin: sa belle couleur jaune paille n'est pas stable après quelques semaines et il s'oxyde rapidement. Ils se sont tournés vers des enseignants-chercheurs de l'Université fédérale de Santa Catarina (UFSC) et de l'Institut de recherche d'État (EPAGRI), qui les ont accompagnés pendant quatre ans.

En 2015, les promoteurs de l'IG affirment que les travaux de la recherche ont fortement contribué à la reconnaissance du vin issu du cépage Goethe ; cela mérite d'être mieux qualifié. Pour ce faire, nous mobilisons la méthode ImpresS, récemment développée au Centre de coopération internationale en recherche agronomique pour le développement (CIRAD), qui s'appuie sur la notion du chemin de l'impact (Douthwaite et al., 2003). Il s'agit d'une démarche participative qui reconstitue d'abord le récit de l'innovation en identifiant les phases, les acteurs clés et leurs interactions. Elle recense les moyens mobilisés (connaissances, financement, expertise...), les produits de la recherche, caractérise l'appropriation de ces produits par des acteurs (résultats), et identifie les divers impacts du processus d'innovation (Barret et al., 2017). Cette méthode met en évidence des liens de causalité entre activités de recherche et impacts. L'article propose de vérifier comment, et à quelle échelle, la recherche agricole a contribué au renforcement des dynamiques collectives dans le territoire, en abordant trois objets et échelles d'analyse: le collectif en charge de l'IG-l'association ProGoethe-, le territoire des «Vales da Uva Goethe», et ses interactions avec les autres territoires. 


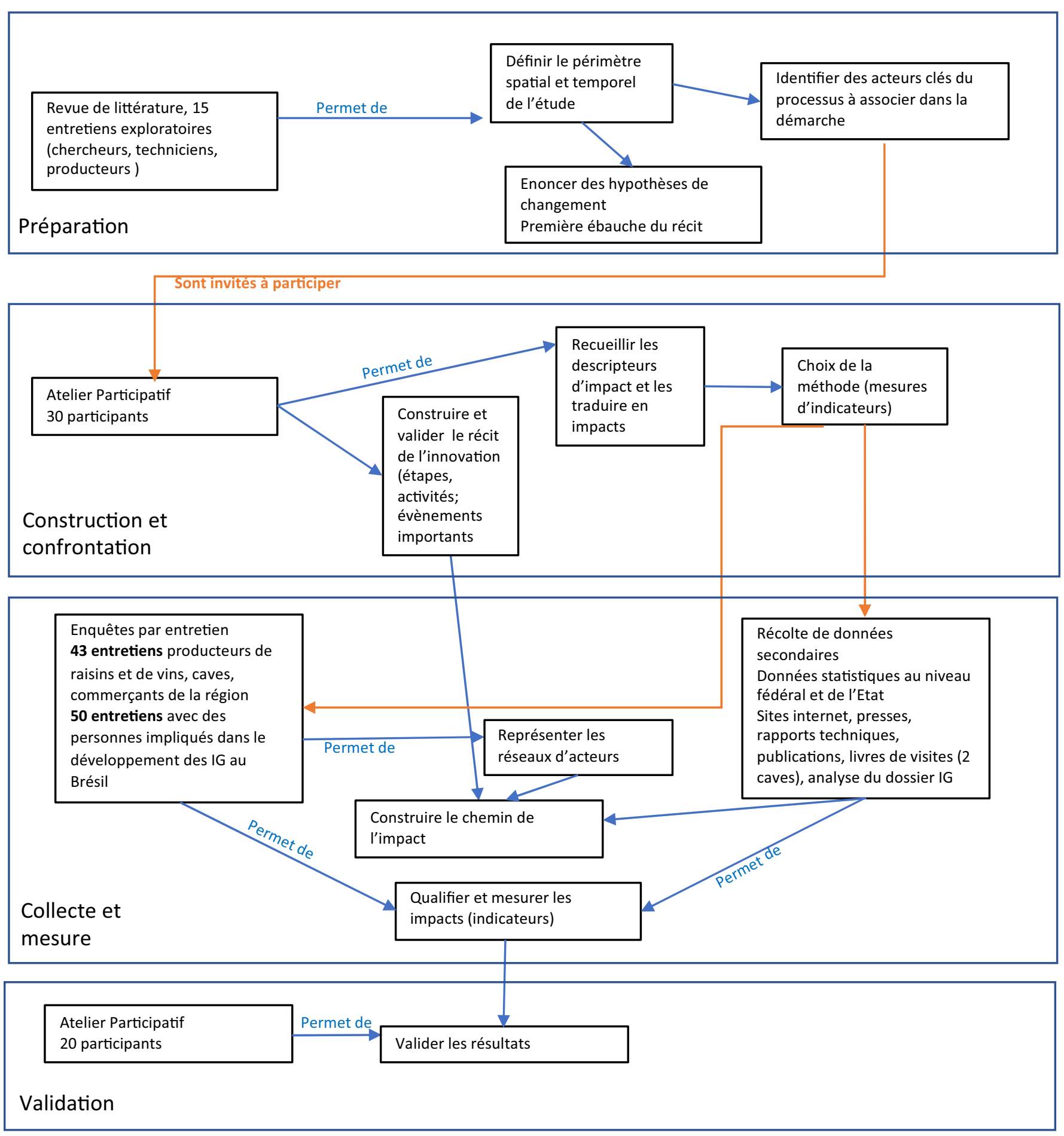

Fig. 2. Principales étapes de la méthode.

Fig. 2. Main steps of the method.

\section{Méthodologie}

La méthode ImpresS s'appuie sur l'utilisation conjointe de quatre outils d'analyse : le récit de l'innovation, la cartographie du réseau d'acteurs, le chemin de l'impact, l'identification et la mesure des impacts et des changements perçus par les acteurs. La méthode ImpresS appliquée à notre cas d'étude est résumée sous forme graphique (Fig. 2). Dans ce cas d'étude, les personnes associées à l'évaluation ont participé aux programmes de recherche-action sur l'IG. Dans une première étape, une revue de littérature et quinze entretiens exploratoires ont permis de définir le périmètre spatial et temporel de l'étude, de poser les premières hypothèses de changement et de faire l'ébauche du récit de l'innovation. Les acteurs clés du processus 
ont été associés à notre démarche. Dans une deuxième étape, un atelier participatif pour identifier les impacts de ce processus a réuni une trentaine de participants (producteurs de vins, personnes impliquées dans le tourisme gastronomique et culturel, représentants d'institutions locales). Ces participants, répartis en petits groupes d'activités (producteurs, consommateurs, chercheurs), ont qualifié collectivement les changements perçus et proposé des méthodes de mesure de ces changements (indicateurs). Au cours de la troisième étape, les données ont été collectées au travers d'une centaine d'entretiens et le recueil des données secondaires (statistiques, sites internet, presse, rapports techniques, publications). Ces informations nous ont permis de construire le chemin de l'impact et de mesurer les impacts. L'étude, initiée en mars 2015, s'est achevée par l'organisation de deux ateliers de validation des résultats, auprès des acteurs du territoire (mars 2016) et auprès des représentants institutionnels (août 2016).

\section{Résultats}

\subsection{L'émergence et le développement de l'indication géographique}

Le périmètre géographique de l'étude couvre neuf municipes (communes) s'étendant sur $3000 \mathrm{~km}^{2}$ (Fig. 1). La période considérée va de 2004 à 2014. Le projet d'IG dans cette région s'inscrit dans une dynamique politique et institutionnelle marquée par la reconnaissance des ressources locales, une revalorisation des liens des produits à leurs lieux d'origine et de nouvelles modalités de promotion des territoires. Dans cette perspective, les acteurs locaux s'organisent au sein d'une association et sollicitent la recherche en vue de la reconnaissance d'une indication géographique pour leur produit emblématique: le vin Goethe. Les organismes de recherche obtiennent le financement de trois projets de recherche successifs entre 2004 et 2009. Cette période est propice à la production de connaissances et aux apprentissages. Plus d'une centaine de publications sont faites en sciences agraires, sciences des aliments, sciences humaines. Le nombre annuel de publications sur le cépage Goethe est multiplié par 10 entre 2007 et 2015 (Cerdan et al., 2016). Le consortium de recherche, constitué de chercheurs, d'étudiants et de producteurs, met en lumière l'histoire de la région (Rebollar et al., 2007) et les spécificités de ce vin, identifie les défauts et teste des innovations pour y remédier. La demande de reconnaissance est déposée en septembre 2009 auprès de l'Institut national de la propriété industrielle (INPI). Commence alors une nouvelle phase. Les maires des municipes concernés par l'IG soutiennent l'initiative en mettant à disposition un local et du personnel. Un bureau d'études spécialisé dans la certification des produits, soutenu par le ministère de l'Agriculture, accompagne les producteurs, définit les modalités de contrôle (plan de contrôle, contrats producteurs) et diffuse la notion d'indication géographique auprès des consommateurs (bandes dessinées, livrets d'informations). Depuis 2014, le vin avec IG est présent sur les marchés des neuf municipes. Les expériences pour améliorer la qualité des vins mettent en avant de nouveaux besoins de recherche. L'arrivée de nouvelles universités (UNESC) et du centre de recherche (EMBRAPA) devrait permettre de consolider l'IG sur des marchés rémunérateurs vers la capitale de l'État du Santa Catarina.

\subsection{Un important processus de recherche-action- formation}

Le chemin de l'impact de l'IG «Vales da Uva Goethe» reflète la rencontre de producteurs de vins avec des chercheurs et des techniciens autour d'un objectif commun, celui de valoriser une ressource locale à travers une IG pour penser des alternatives de développement pour le territoire. Avant d'analyser les différentes relations de causalité entre la recherche et les effets observés, nous proposons de revenir sur les différentes catégories du chemin de l'impact (Fig. 3). Les moyens mobilisés dans le processus d'innovation sont des connaissances génériques des chercheurs (sur les démarches de qualité, sur la conduite de la vigne) et des connaissances spécifiques des producteurs sur le cépage. Des moyens financiers et des infrastructures (instruments de mesure, stations météorologiques, laboratoires) sont mis à disposition. Un dernier input important est la présence de l'association des producteurs, créée en 2005, qui sera un acteur central dans le processus d'innovation. Les projets de recherche livrent une large gamme de produits. Les nouvelles connaissances, issues des expérimentations sur les modes de production et de vinification, aident les producteurs à élaborer leur cahier des charges. La délimitation de l'aire et la démonstration du lien au territoire se basent sur des éléments historiques et des données pédoclimatiques localisées (Rebollar et al., 2007; Back et al., 2012). Les leçons apprises de cette démarche motivent les chercheurs à concevoir une formation à distance destinée aux cadres en charge de développer des signes indicateurs de la qualité. Les résultats regroupent l'ensemble des initiatives et des dispositifs qui contribuent à la diffusion des produits de la recherche. Les producteurs mobilisent les connaissances acquises pour produire un livre sur les «Vales da Uva Goethe», le discours sur les spécificités du produit est régulièrement présenté dans les événements viticoles. La capacité à innover et à interagir est un autre résultat du processus d'innovation. Les producteurs sont en mesure d'identifier de nouveaux problèmes de recherche, de dialoguer avec la recherche pour monter de nouveaux projets ou de se mobiliser pour obtenir des ressources humaines supplémentaires (à l'exemple du recrutement du chercheur en œnologie évoqué plus bas). Les impacts observés concernent les acteurs impliqués dans le processus d'innovation (Impact 1) et indirectement les acteurs de la région ou du pays (Impact 2). Les personnes interrogées relèvent une professionnalisation des producteurs, constatent le développement de nouveaux marchés avec notamment la croissance de la vente directe et de l'œnotourisme. Elles soulignent le phénomène de revalorisation de l'activité vinicole dans la région qui bénéficie aux producteurs de vins IG mais aussi aux autres petits producteurs de vins artisanaux élaborés avec d'autres variétés. Avant 2004, les vins de la région étaient de qualité très irrégulière et peu valorisés par les habitants et les établissements touristiques de la région.

\subsection{Un processus d'innovation complexe}

Le chemin de l'impact de l'IG reflète le caractère complexe du processus d'innovation par la présence de plusieurs sousensembles «moyen, produit, résultat, impact». Dans un souci de clarté, nous avons privilégié d'analyser ici trois sous-ensembles du chemin de l'impact en les présentant séparément (Fig. 4). 
Moyens

Produits

Résultats

Impacts 1

Impacts 2

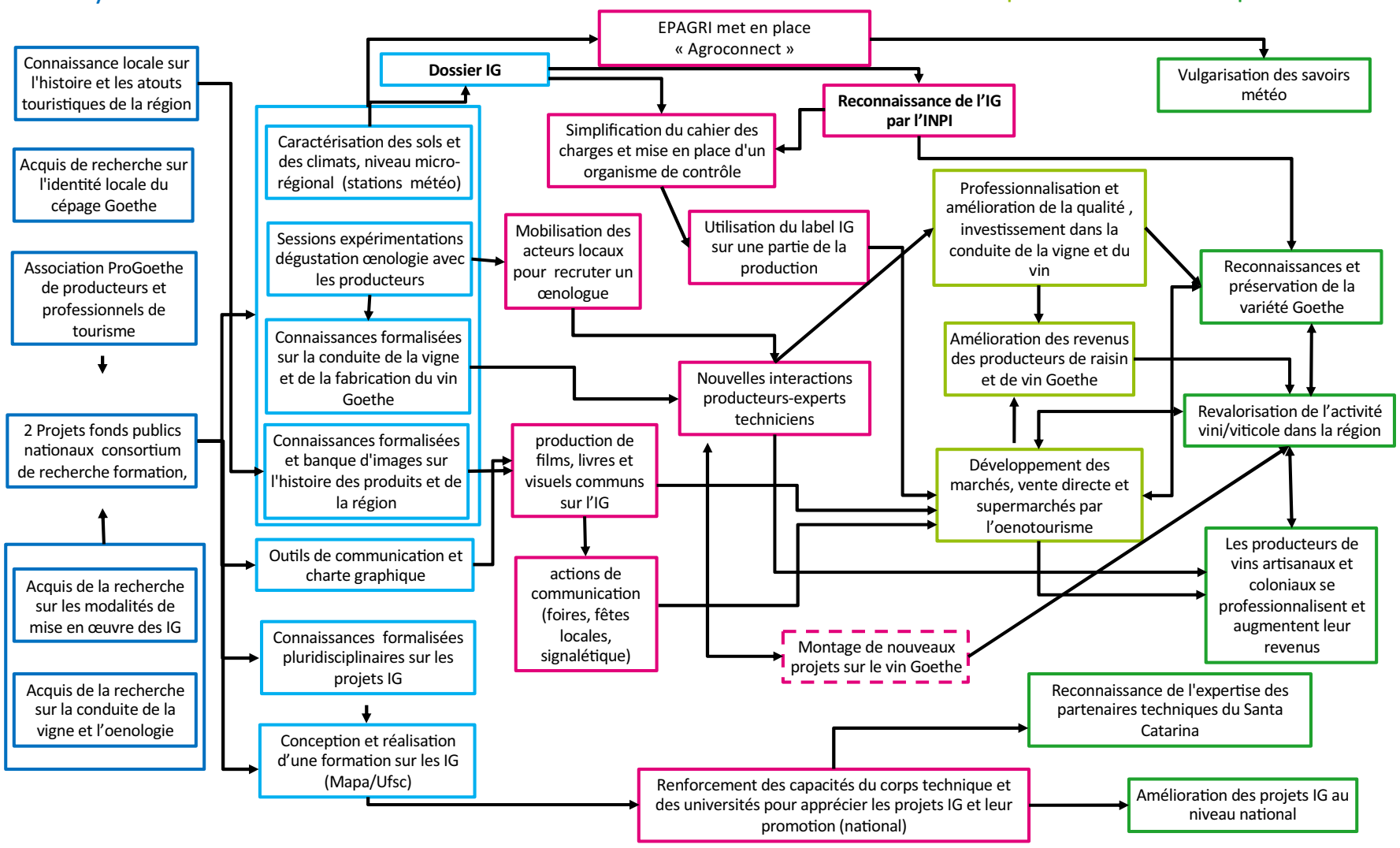

Fig. 3. Chemin de l'impact de l'IG "Vales da Uva Goethe».

Fig. 3. Impact pathway of the GI "Vales da Uva Goethe".

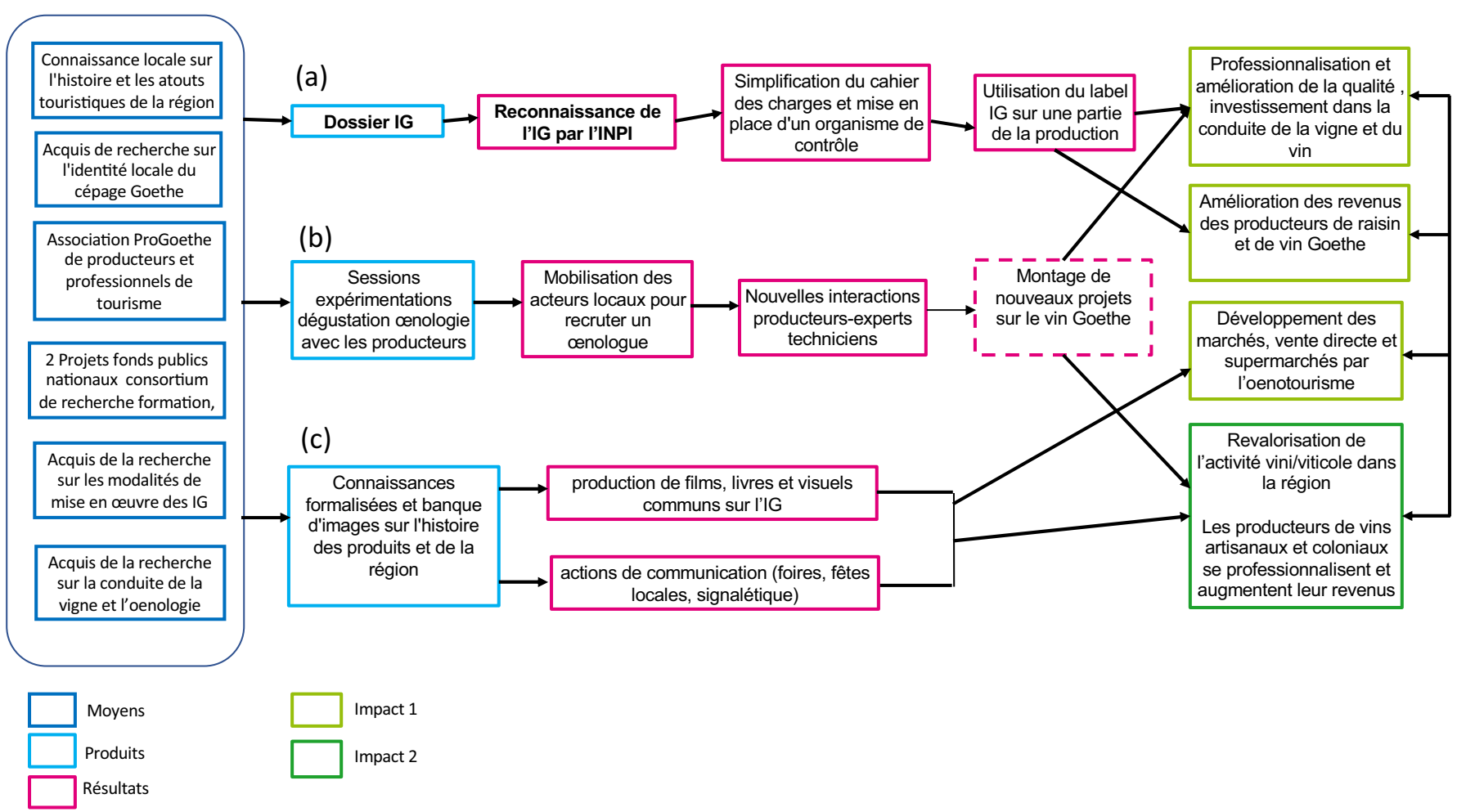

Fig. 4. Sélection de trois sous-ensembles tirés du chemin de l'impact.

Fig. 4. Selection of three sub-group from the impact pathways. 
Le premier sous-ensemble (a) concerne la demande d'enregistrement auprès de l'INPI. Le dossier de demande d'IG mobilise des produits issus des recherches et des innovations techniques. Il s'agit d'un document très complet mais difficile à contrôler et à mettre en œuvre. La révision et la simplification du cahier des charges par les producteurs ont facilité son adoption. Ces résultats contribuent à trois impacts majeurs pour les opérateurs: une professionnalisation de la production de vin dans la région qui permet d'obtenir des vins de meilleure qualité, la création de nouveaux produits (vins mousseux), le développement de nouveaux marchés.

Le deuxième sous-ensemble (b) porte sur les sessions de dégustation collective. Au-delà des changements de pratiques culturales ou vinicoles qu'elles ont rendus possibles, ces séances de travail ont permis la mobilisation des producteurs pour le recrutement d'un chercheur œnologue dans la région (professionnalisation). De plus, les échanges entre les différentes parties prenantes au cours de ces sessions favorisent l'émergence d'un climat de confiance et d'un langage commun entre chercheurs et producteurs. Ceci se révèle important pour le futur de l'IG, il renforce la capacité des acteurs à innover et à monter de nouveaux projets de recherche.

Le troisième sous-ensemble (c) est le fait des promoteurs de l'IG qui publient des livres, des bandes dessinées ou des prospectus. Ces initiatives contribuent au développement des marchés (ventes directes) et à la revalorisation du vin dans la région. Ici, les chercheurs ne sont pas impliqués.

\subsection{La contribution de la recherche au renforcement des dynamiques collectives}

Nous proposons, à l'aide du chemin de l'impact, de vérifier comment et à quelle échelle la recherche agricole a contribué au renforcement des dynamiques collectives dans le territoire. Nous faisons l'hypothèse que cette contribution peut varier en fonction de l'échelle d'analyse. Nous aborderons successivement trois échelles distinctes: le collectif en charge de l'IG-l'association ProGoethe-, le territoire des «Vales da Uva Goethe », et les interactions avec les autres territoires.

\subsubsection{L'association ProGoethe}

L'association regroupe des producteurs de raisins et de vins qui, jusqu'alors, n'avaient pas l'habitude de travailler ensemble. Les uns et les autres se connaissaient peu. La démarche de recherche-action proposée par les chercheurs, qui associe l'ensemble des parties prenantes, a été cruciale. Les membres de l'association ont activement participé à l'élaboration des projets (demandes de financements) et aux suivis des activités. Il est important de souligner qu'au départ, les chercheurs, les techniciens et les viticulteurs sont tous des apprenants. Les producteurs connaissent leur raisin mais ne maîtrisent pas les nouvelles technologies de vinification, les chercheurs les maîtrisent mais n'ont jamais travaillé avec le cépage Goethe. Cela occasionnera quelques essais-erreurs mémorables comme l'explosion de bouteilles dans les laboratoires et les caves (Ferreira-Lima et al., 2013). Cette période propice à la production de connaissances et aux apprentissages laisse une large place aux dialogues et aux échanges entre les producteurs, les chercheurs et les techniciens. Au fil des discussions, les associés construisent une vision commune de
l'IG potentielle et redéfinissent les missions de leur association. L'émergence d'une dynamique collective au sein de cette association permet d'élaborer le cahier des charges et les règles de contrôles et d'organiser la défense et la promotion de leur produit. L'obtention du recrutement d'un chercheur en œnologie dans la région conforte les producteurs dans l'idée que l'association peut intercéder auprès des principaux représentants des institutions publiques «Depuis, on se sent plus forts, on nous a entendus » affirme un producteur (entretien 2015). Cette association est toutefois mise à l'épreuve. Le vin issu de cépage Goethe ne répond pas aux critères de qualité du discours dominant porté par les producteurs de vins d'autres régions du Brésil. L'apport de connaissances formalisées et l'implication de chercheurs lors de tables de négociation se sont avérés utiles pour la reconnaissance du cépage Goethe face à ses concurrents. La contribution de la recherche est relativement claire dans ce premier niveau d'analyse. Il s'agit de produire de nouvelles connaissances, formaliser les connaissances scientifiques et les savoirs locaux, accompagner les acteurs dans leurs prises de décision.

\subsubsection{La recherche, I'IG et le territoire}

Bien que centrées sur l'association ProGoethe, les activités de recherche ont impacté le territoire d'une façon plus large. Nous le montrerons à partir de trois illustrations.

La première concerne l'émergence d'un nouveau territoire, les «Vales da Uva Goethe». L'aire géographique de production des vins avec IG couvre en effet plusieurs municipes qui ont jusqu'alors plutôt l'habitude de rivaliser entre eux, chacun ayant une vision et une histoire différentes avec le vin issu de cépage Goethe. Dans les documents historiques, ce sont les villes d'Urussanga et de Pedra Grande qui sont signalées comme régions productrices du vin. La dénomination «Vales da Uva Goethe » n'existe pas. Le choix de cette dénomination souligne la volonté de regrouper l'ensemble des personnes ayant contribué à l'histoire du vin de cépage Goethe sans qu'un municipe ne soit davantage mis en avant qu'un autre. Un des défis majeurs pour l'association ProGoethe est que l'ensemble des acteurs des municipes (les producteurs mais aussi les commerçants, les restaurateurs, les conseillers municipaux) se reconnaissent dans cette vision territoriale des «Vales da Uva Goethe» et la défendent. Ces initiatives sont surtout le fait des acteurs locaux (privés mais aussi politiques), mais la recherche y a aussi contribué en légitimant le bien-fondé de la démarche à partir de témoignages d'autres expériences vécues ailleurs et en accompagnant les producteurs dans leur démarche collective.

La deuxième illustration concerne la revalorisation des activités vitivinicoles. Les acteurs affirment qu'elle est aujourd'hui bien engagée dans la région: des producteurs se lancent, des jeunes reprennent l'exploitation ou l'entreprise familiale, et tous sont fiers de leur produit et de leur région. La reconnaissance du cépage Goethe est un élément fédérateur dans ce processus de valorisation de l'activité vitivinicole dans la région. Ce nouvel intérêt est le fait des acteurs locaux mais aussi de la recherche par la mise à disposition de connaissances issues du processus d'interactions que les acteurs ont su valoriser (livres, photos, dépliants).

Enfin, le recrutement d'un chercheur œnologue par l'institution de recherche de l'État pour une affectation dans 
la zone de production et son implication dans l'expérimentation participative et la formation ( $60 \%$ de son temps) jouent également un rôle fondamental. Sa disponibilité et sa proximité avec le monde paysan, lui-même étant fils de viticulteur d'une autre région du Santa Catarina, contribuent à l'instauration d'un climat de confiance. Ainsi, les acteurs locaux et l'œnologue contribuent à la diffusion des connaissances et des résultats observés au cours du projet, au-delà de ses bénéficiaires directs : usage de levures et de l'enzyme Beta-Glycosidase par exemple (Bonet et al., 2015). Aujourd'hui, les producteurs de vin artisanal ne prétendent pas certifier leur production mais améliorent la qualité de leur produit et participent aux actions collectives qui valorisent la vitiviniculture de la région (foires, marchés, fêtes, accueil touristique, ventes directes...).

\subsubsection{Les rôles des réseaux d'experts et des mouvements sociaux dans les connexions externes}

La troisième contribution de la recherche au renforcement de l'action collective dans les territoires concerne les relations et la capacité des acteurs du territoire à interagir avec d'autres producteurs de régions différentes. Cette contribution se traduit dans le chemin de l'impact par les résultats «Nouvelles interactions producteurs-experts techniciens » et « Montage de nouveaux projets sur le vin issu de cépage Goethe». Une situation favorable s'établit peu à peu pour développer de nouveaux partenariats et des interconnexions avec d'autres territoires. La recherche joue ici un rôle de médiateur entre l'association ProGoethe et des réseaux nationaux mobilisés sur les mêmes thématiques. Wilkinson et al. (2017) ont souligné le rôle décisif des réseaux sociaux dans le développement et la promotion des IG et des produits d'origine au Brésil en distinguant le réseau d'experts et les mouvements sociaux que nous considérons comme un réseau de mobilisation (Wilkinson et al., 2017). Le réseau d'experts est constitué de chercheurs et de cadres techniques. Son activité consiste à organiser des séminaires, créer une liste de discussion, concevoir des modules de formation, en présentiel ou à distance. L'association ProGoethe, l'UFSC, Le CIRAD et l'EPAGRI contribuent à ces différentes activités. Aujourd'hui l'IG «Vales da Uva Goethe » est reconnue et des personnes intéressées par les IG leur rendent souvent visite. Au côté de ce réseau d'experts, le réseau de mobilisation regroupe des organisations de la société civile, des chefs cuisiniers ou des représentants de mouvements sociaux internationaux (Slow Food). L'implication des producteurs à ce deuxième réseau a motivé l'association pour la défense et la reconnaissance du cépage hybride Goethe. Elle a permis de connecter les acteurs de cette IG à d'autres régions et d'autres pays portés par le même intérêt de valoriser les ressources locales pour un développement durable. La participation de plusieurs acteurs du territoire des "Vales da Uva Goethe» et de l'association au salon Terra Madre à Turin (Italie) a conforté les acteurs dans leur projet de territoire.

\section{Discussion: quelle recherche pour accompagner les acteurs dans leurs démarches de qualité ?}

Le cas de l'IG «Vales da Uva Goethe» est une bonne illustration des impacts potentiels d'une IG dans un territoire donné. Nous observons des éléments connus dans la littérature sur le développement de nouveaux marchés et l'amélioration de la qualité des produits (Vandecandelaere et al., 2010). La méthode ImpresS et le chemin de l'impact contribuent à la réflexion en identifiant les impacts et en confirmant l'importance des programmes de recherche-action-formation dans la mise en place des IG. Ceci nous invite à mieux spécifier le rôle et l'implication de la recherche dans ces processus d'innovation. L'implication de la recherche évolue dans le temps et dans ses modalités d'action. Nous avons analysé dans cet article les contributions possibles de la recherche aux renforcements des dynamiques collectives. Les projets à l'initiative des producteurs conduisent à l'émergence d'un consortium multi-acteurs pluridisciplinaires associant des chercheurs des sciences du sol, de l'œnologie, de l'agronomie, et des sciences humaines. Une fois le dossier remis aux autorités compétentes, les projets de recherche s'achèvent et ce consortium bâti pour l'élaboration du dossier se défait, les chercheurs se retirent. Quelques producteurs contactent ensuite les chercheurs par téléphone de façon informelle, en quête de conseils techniques. Ces mêmes producteurs ont toutefois été très critiques sur le retrait des chercheurs, qu'ils ont vécu comme un abandon pour plusieurs raisons. D'abord, les résultats des expériences mettent en évidence un besoin de recherche et de connaissances nouvelles supplémentaires sur la conduite et le comportement de ce cépage hybride dans la région. Ensuite, la présence de la recherche au côté des producteurs joue un rôle de garant et donne une forte crédibilité aux initiatives engagées par les producteurs. Une autre dimension apportée par la recherche est le renforcement des compétences des acteurs locaux et à travers la formation. De nombreuses situations d'apprentissage ont pu être décrites et identifiées dans le chemin de l'impact. Plus que l'obtention d'une IG, ces projets ont développé la capacité à innover des acteurs (Douthwaite et Hoffecker, 2017). Ces derniers semblent aujourd'hui en mesure de développer de nouveaux projets, d'améliorer la qualité de leurs produits et de répondre aux demandes des marchés. Ces observations rejoignent Lardon (2009) et les travaux développés en économie de la connaissance qui considèrent la formation comme élément médiateur entre la recherche et l'action. Nous sommes en présence de processus d'apprentissage capables de susciter des dynamiques de changement et de développement territorial (Foray, 2000; Chia et al., 2007; Coudel, 2008).

\section{Conclusion}

Cet article analyse les possibles contributions de la recherche dans la mise en œuvre d'une demande de reconnaissance d'un produit d'origine par une IG et le renforcement des actions collectives d'un territoire donné. Trois échelles d'analyse sont mobilisées et confirment que la recherche agricole, sous certaines conditions, est à même d'appuyer les dynamiques collectives dans les territoires. La recherche contribue à produire et à formaliser de nouvelles connaissances pour les promoteurs de l'IG. Les méthodes de recherche-expérimentation-formation associant les partenaires économiques se révèlent déterminantes pour faciliter les échanges entre producteurs et chercheurs. Ces méthodes améliorent les impacts de la recherche en les organisant pour 
devenir une contribution aux dynamiques de développement local. Elles permettent d'instrumenter les démarches de qualification en aidant à co-construire les repères et en veillant à les rendre appropriables. L'intégration de l'IG dans le projet d'un territoire est un défi plus difficile à mettre en œuvre. La définition des aires de production conduit à faire bouger les frontières et à construire de nouveaux territoires (Pecqueur, 1996). Cette construction est surtout du ressort des acteurs locaux (privés et politiques). Mais la recherche peut y contribuer en apportant des garanties et une certaine légitimité. Le troisième niveau de contribution porte sur la capacité des chercheurs à établir de nouvelles interrelations avec d'autres territoires. Nous nous sommes volontairement centrés sur deux types de réseaux supra-territoriaux, le réseau d'experts, essentiellement porté par des chercheurs, et le réseau de mobilisation, porté par les mouvements sociaux. Pour chaque étape, la contribution de la recherche évolue. Elle va souvent au-delà des impacts initialement formulés par les chercheurs dans leur projet de recherche. La méthode ImpresS produit elle-même des effets. Elle se révèle une étape importante où les acteurs d'un processus d'innovation (promoteurs de l'IG et chercheurs) engagent une réflexion sur les manières de produire des connaissances scientifiques pour les rendre «actionnables» (Béguin et Cerf, 2009).

\section{Références}

Allaire G. 2009. Diversité des indications géographiques et positionnement dans le nouveau régime de commerce international. In: Tekelioglu Y, Ilbert H, Tozanli S, eds. Les produits de terroir, les indications géographiques et le développement local durable des pays méditerranéens. Montpellier: CIHEAM, pp. 53-65.

Back ÁJ, Vieira HJ, Dela Bruna E. 2012. Tendências climáticas e produção de uva na região dos Vales da Uva Goethe. Pesquisa Agropecuária Brasileira 47: 497-504.

Barret D, Blundo-Canto G, Dabat MH, Devaux-Spatarakis A, Faure G, Hainzelin E, et al. 2017. Guide méthodologique ImpresS. Évaluation ex-post des impacts de la recherche agronomique dans les pays du Sud. Montpellier: CIRAD. Disponible sur http:// agritrop.cirad.fr/586223.

Béguin P, Cerf M (Dir.). 2009. Dynamique des savoirs, dynamique des changements. Toulouse: Octarès Éditions, 306 p.

Bonet J, Agustini BC, Morini MAL, Silva GA. 2015. Caracterização de leveduras isoladas de bagas de uva Goethe da região de Urussanga (SC) para a elaboração de vinhos. In: Anais do XV Congresso Latino-Americano de Viticultura e Enologia e XIII Congresso Brasileiro de Viticultura e Enologia, Bento Goncalves, Brasil.

Boisvert V. 2013. Marchandisation ou patrimonialisation? L'économie de la biodiversité en perspective In: Juhé-Beaulaton D, Cormier-Salem MC, de Robert P, Roussel B, eds. Effervescence patrimoniale au Sud. Entre nature et société. Montpellier: IRD éditions, collection Latitudes, pp. 27-54.

Cerdan C, Andrade NG, Velloso CQ, Silva AL,Vieira HJ. 2016. Impactos da pesquisa na Indicação de Procedência (IP) Vales da Uva Goethe em Santa Catarina. In: Liliana Locatelli. (Org.).
Indicações Geográficas: desafiose perspectivas nos 20 anos da Lei de Propriedade Industrial. Rio de Janeiro: Lumen Juris, vol. 1, pp. 69-88.

Chia E, Piraux M, Dulcire M. 2007. Apprentissage et gouvernance territoriale: quelles relations? Le cas des CTE à la Réunion, $47^{\circ}$ colloque international de l'ERSA, Paris, 29 août-2 septembre 2007.

Coudel E. 2008. Which competencies and learning facilitate the involvement of local actors in territorial governance? The example of a Farmer University in Brazil. International Journal of Sustainable Development 11(2-4): 206-225.

Douthwaite B, Hoffecker E. 2017. Towards a complexity-aware theory of change for participatory research programs working within agricultural innovation systems. Agricultural Systems 155: 88-102.

Douthwaite B, Kuby T, Van de Fliert E, Schulz S. 2003. Impact pathway evaluation: an approach for achieving and attributing impact in complex systems. Agricultural Systems 78(2): 243-265.

Ferreira-Lima NE, Burin VM, Bordignon-Luiz MT. 2013. Characterization of Goethe white wines: Influence of different storage conditions on the wine evolution during bottle aging. European Food Research and Technology 237(4): 509-520.

Foray D. 2000. L'économie de la connaissance. Repères, vol. 302. Paris : Éditions La Découverte, 124 p.

Fournier S. 2016. Évaluer ex ante l'impact d'une indication géographique? De la difficulté d'appréhender des processus d'action collective et d'innovation au sein de systèmes complexes In: Économies et Sociétés, Série Systèmes Agroalimentaires. vol. 37. Paris : Isméa Les Presses, pp. 1167-1184.

Lardon S. 2009. Former des ingénieurs-projets en développement territorial. Un itinéraire méthodologique pour faciliter la participation des acteurs. In: Béguin P, Cerf M (dir.) Dynamiques des savoirs, dynamiques des changements. Toulouse : Octarès Éditions, pp. 209-227.

OMC. 1994. Aspects des droits de propriété intellectuelle qui touchent au commerce. Partie II - Normes concernant l'existence, la portée et l'exercice des droits de propriété intellectuelle. Section 3 : Indications géographiques. Marrakech, OMC. Disponible sur https://www.wto. org/french/docs_f/legal_f/27-trips_04b_f.htm\#indications.

Pecqueur B. 1996. Dynamiques territoriales et mutations économiques. Paris : l'Harmattan, 246 p.

Rebollar P, Velloso C, Ern R, Vieira H, Silva A. 2007. Vales da uva Goethe. Urussanga (Brasil): PROGOETHE, 64 p.

Sylvander B, Isla A, Wallet F. 2011. Under what conditions geographical indications protection schemes can be considered as public goods for sustainable development? In: Torre A, Traversac JB, eds. Territorial governance, local development, rural areas and agrofood systems. Heidelberg (Germany): Physica Verlag.

Torre A, Wallet F (eds.). 2016. Regional development in rural areas. Analytical tools and public policies. Berlin: Springer, 110 p.

Vandecandelaere E, Arfini F, Belletti G, Marescotti A (eds.). 2010. Linking people, places and products: A guide for promoting quality linked to geographical origin and sustainable geographical indications. Rome: FAO, 193 p.

Wilkinson J, Cerdan C, Dorigon C. 2017. Geographical indications and origin products in Brazil. The interplay of institutions and networks. World Development 98: 82-92.

Citation de l'article : Cerdan C, Andrade N, Lima da Silva A, Vieira H, Silva E, Le Guerroué JL. 2018. La recherche agricole, vecteur de dynamiques collectives des territoires ruraux: 1'Indication géographique «Vales da Uva Goethe» au Brésil. Cah. Agric. $27: 25007$. 